\title{
The shortwave radiative forcing bias of liquid and ice clouds from MODIS observations
}

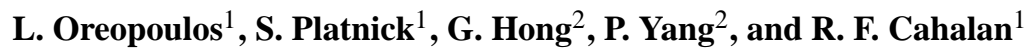 \\ ${ }^{1}$ Laboratory for Atmospheres, NASA-GSFC, Greenbelt, MD, USA \\ ${ }^{2}$ Dept. of Atmospheric Sciences, Texas A\&M University, College Station, TX, USA
}

Received: 16 March 2009 - Published in Atmos. Chem. Phys. Discuss.: 28 April 2009

Revised: 3 August 2009 - Accepted: 10 August 2009 - Published: 19 August 2009

\begin{abstract}
We present an assessment of the plane-parallel bias of the shortwave cloud radiative forcing (SWCRF) of liquid and ice clouds at $1 \mathrm{deg}$ scales using global MODIS (Terra and Aqua) cloud optical property retrievals for four months of the year 2005 representative of the meteorological seasons. The (negative) bias is estimated as the difference of SWCRF calculated using the Plane-Parallel Homogeneous (PPH) approximation and the Independent Column Approximation (ICA). PPH calculations use MODISderived gridpoint means while ICA calculations use distributions of cloud optical thickness and effective radius. Assisted by a broadband solar radiative transfer algorithm, we find that the absolute value of global SWCRF bias of liquid clouds at the top of the atmosphere is about $6 \mathrm{~W} \mathrm{~m}^{-2}$ for MODIS overpass times while the SWCRF bias for ice clouds is smaller in absolute terms by about $0.7 \mathrm{~W} \mathrm{~m}^{-2}$, but with stronger spatial variability. If effective radius variability is neglected and only optical thickness horizontal variations are accounted for, the absolute SWCRF biases increase by about $0.3-0.4 \mathrm{~W} \mathrm{~m}^{-2}$ on average. Marine clouds of both phases exhibit greater (more negative) SWCRF biases than continental clouds. Finally, morning (Terra)-afternoon (Aqua) differences in SWCRF bias are much more pronounced for ice clouds, up to about $15 \%$ (Aqua producing stronger negative bias) on global scales, with virtually all contribution to the difference coming from land areas. The substantial magnitude of the global SWCRF bias, which for clouds of both phases is collectively about $4 \mathrm{~W} \mathrm{~m}^{-2}$ for diurnal averages, should be considered a strong motivation for global climate modelers to accelerate efforts linking cloud schemes capable of subgrid condensate variability with appropriate radiative transfer schemes.
\end{abstract}

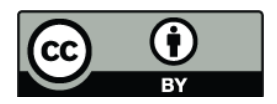

Correspondence to: L. Oreopoulos (lazaros.oreopoulos@nasa.gov)

\section{Introduction}

In a recent study Oreopoulos et al. (2007) examined the albedo bias due to use of MODIS (Moderate Resolution Imaging Spectroradiometer) $1^{\circ} \times 1^{\circ}$ gridpoint means of liquid cloud optical thickness and effective radius instead of accounting for their underlying distributions. This so-called Plane-Parallel Homogeneous (PPH) bias (Cahalan et al., 1994a) remains mostly unaddressed in the solar radiation calculations of Large Scale Models even though it is a known source of systematic error that has to be compensated by underprediction of other cloud properties such as cloud condensate and cloud fraction. The PPH albedo values of $\sim 0.03$ found in Oreopoulos et al. (2007), i.e., approaching $10 \%$ of the proper liquid cloud albedo with spatial cloud variations included, reaffirmed the importance of horizontal cloud variability for accurate assesments of the solar radiation budget. The other motivation behind that study was the lack of an extensive global mapping of the PPH bias, with previous published satellite studies being mainly assessments of the visible PPH bias over limited parts of the globe (Barker 1996, Oreopoulos and Davies 1998; Pincus et al., 1999), or focusing primarily on parameters quantifying the underlying cloud horizontal inhomogeneity (Rossow et al., 2002; Oreopoulos and Cahalan, 2005) instead of the broadband bias itself.

The current study seeks to expand the Oreopoulos et al. (2007) study by, among others, providing better seasonal coverage (a representative month for each season is used instead of a winter/summer only month) and by also including clouds classified by MODIS to be of ice phase (near their top). A newer, improved version of MODIS cloud data is used, and emphasis is placed on the PPH bias of the shortwave (SW) Cloud Radiative Forcing (SWCRF), where the forcing is defined as the difference in SW irradiance measured for average atmospheric conditions and that measured in the absence of clouds for the same region and time period.

Published by Copernicus Publications on behalf of the European Geosciences Union. 
The advantage of examining forcing instead of albedo bias is that the former takes into account the areal coverage and frequency of occurrence of liquid and ice clouds and directly links the magnitude of the bias to the radiative energy budget. The SWCRF bias features presented here along with the online collection of PPH albedo biases from ISCCP (International Satellite Cloud Climatology Project) at http://isccp.giss.nasa.gov (from larger reference areas and assuming a different cloud classification of low, middle and high clouds) provides a fairly comprehensive picture of the radiative effects of horizontal cloud inhomogeneity. Global models that aspire to produce clouds with subgrid variability, and super-parameterization approaches (Khairoutdinov et al., 2005) should find these datasets useful in validation studies.

The paper is organized as follows: Computational and dataset information are provided in the next section; the various dependences of the global and local SWCRF biases are detailed in the five subsections of Sect. 3, while conclusions, along with suggestions on how to exploit the results for global model validation, are provided in Sect. 4.

\section{Dataset and radiative transfer calculations}

As in Oreopoulos et al. (2007), we use daily MODIS Level3 ( $1^{\circ}$ gridded) daytime data from both the Terra $(\sim 10: 30$ local time overpass) and Aqua ( $\sim 13: 30$ overpass) satellites (datasets MOD08_D3 and MYD08_D3, respectively). Here, we use products from the most recent processing stream, Collection 5, and extend the study to four months, January, April, July, and October 2005. We extract the mean solar zenith angle (SZA), and separately for each cloud phase (liquid/ice) the mean daily values of column-integrated optical thickness $(\bar{\tau})$, effective radius $\left(\bar{r}_{e}\right)$, cloud fraction of successful cloud retrievals $\left(A_{c}\right)$, as well as one-dimensional (1-D) histograms of $\tau$ and joint (2-D) histograms of $\tau-r_{e}$ and $\tau$ cloud top temperature $\left(T_{c}\right)$, constructed by sampling every 5 th pixel of the original $1 \mathrm{~km}$ resolution retrieval (King et al., 2003). The 1-D histograms of $\tau$ are resolved in 45 bins for liquid clouds and 30 bins for ice clouds. The 2-D histograms of $\tau$ and $r_{e}$ are resolved in 110 bins (11 for $\tau$ covering the range $0.1-100$ and 10 for $r_{e}$ covering the range 3 to $30 \mu \mathrm{m}$ ) for liquid clouds, and 143 bins for ice clouds (11 for $\tau$ covering the range $0.1-100$ and 13 for $r_{e}$ covering the range 5 to $90 \mu \mathrm{m}$ ); the joint histograms of $\tau$ and $T_{c}$ are resolved in 143 bins ( 11 for $\tau$ and 13 for $T_{c}$ ) for both phases. Statistics for clouds assigned the "undetermined" phase are not used. Except for high latitudes where gridpoints can be revisited within the same day due to orbital swath overlap, for the largest portion of the globe the daily histograms represent mainly instantaneous spatial variability of $\tau$ and $r_{e}$ within the $1^{\circ}$ gridpoint. The lower number of instantaneously viewed pixels within $1^{\circ}$ gridpoints at high latitudes is balanced by multi-sampling from revisits within the same day, making thus the daily cloud variability spatiotemporal in nature instead of purely spatial. The consequences for global model validation of the constant $1^{\circ}$ grid of the MODIS Level-3 dataset and the MODIS sampling strategy is discussed later.

The radiative transfer calculations yielding daily atmospheric column albedo are performed with a version of the broadband CLIRAD-SW radiation model of Chou et al. (1998). The salient features of this algorithm and the manner in which it is interfaced with the MODIS retrievals, Global Data Assimilation System (GDAS) atmospheric information (Derber et al., 1991) and MODIS-derived surface albedo (Moody et al., 2005) are described in Oreopoulos et al. (2007) and Oreopoulos and Platnick (2008). In our radiative transfer calculations, clouds are placed in the layer whose top temperature is closest to the mean cloud top temperature $\left(\bar{T}_{c}\right)$ as derived from the joint histogram of $\tau$ and $T_{c}$. The mean cloud top temperature was derived from joint histograms because the corresponding Level-3 mean cloud product does not distinguish between liquid and ice phases. Since the MODIS-inferred cloud properties are assigned to a single layer within our atmospheric profile, there is no need to treat cloud overlap which is not resolved anyway by the passive MODIS observations.

An important modification in our version of CLIRAD-SW is the introduction of a new method of calculating cloud optical properties (extinction, single-scattering albedo, asymmetry factor). The changes implemented for liquid clouds are described in Oreopoulos et al. (2007) and Oreopoulos and Platnick (2008). One of the reasons the original parameterization was changed was to extend its applicable range above the upper limit of $20 \mu \mathrm{m}$ for which it was designed originally, given that MODIS liquid effective radius retrievals can be as high as $30 \mu \mathrm{m}$. The retrieved $\tau$ from MODIS was used in the ultraviolet-visible (UV-VIS) spectral region of CLIRADSW which assumes a constant cloud extinction coefficient. The spectral values of $\tau$ for the remaining three spectral regions in the solar infrared (also with constant extinction coefficients within their bounds) were calculated by rescaling the MODIS $\tau$ with the ratio of the extinction coefficient for those bands to its counterpart in the UV-VIS band for the appropriate value of $r_{e}$ provided by the retrievals.

For ice clouds a new parameterization of scattering properties is used, based on the ice particle single-scattering properties of Yang et al. $(2000,2005)$. The ice habit distribution is consistent with that used for the MODIS retrieval look-up tables of Collection 5, which comes from Baum et al. (2005). The particle size distributions of ice clouds come from several compaigns (see details in Baum et al., 2005) and from 21 of the 30 distributions in $\mathrm{Fu}$ (1996). The cloud mass extinction coefficient $(\beta)$, single scattering co-albedo $(1-\varpi)$ and asymmetry factor $(g)$ are fitted as a function of the effective ice crystal diameter $D_{e}$ of the particle size distribution as follows:

$\beta=a_{0}+\frac{a_{1}}{D_{e}}+\frac{a_{2}}{D_{e}^{2}}$ 
$1-\bar{\omega}=b_{0}+b_{1} D_{e}+b_{2} D_{e}^{2}+b_{3} D_{e}^{3}+b_{4} D_{e}^{4}+b_{5} D_{e}^{5}$

$g=\left\{\begin{array}{l}c_{0}+c_{1} D_{e} \text { for } D_{e} \leq 40 \mu \mathrm{m} \text { and } D_{e} \geq 200 \mu \mathrm{m} \\ c_{0}+c_{1} D_{e}+c_{2} D_{e}^{2}+c_{3} D_{e}^{3}+c_{4} D_{e}^{4}+c_{5} D_{e}^{5} \text { for } 40 \mu \mathrm{m}<D_{e}<200 \mu \mathrm{m}\end{array}\right.$

where the fitting coefficients $a_{0}, a_{1}, a_{2}, b_{0}, b_{1}$, etc., are found from regressions. One set of coefficients is available for each solar infrared spectral region (i.e., constant single-scattering properties are again assumed within these spectral regions) and 8 sets of coefficients for the UV-VIS spectral region, one for each of its 8 bands. Thus, there are 11 sets of fitting coefficients in total. The ice optical thickness retrieved by MODIS was assigned to band 8 which covers the visible portion of the model's UV-VIS spectral region. The optical thicknesses in the remaining model bands and spectral regions were found using the same rescaling procedure described above for liquid clouds.

Similar to Oreopoulos et al. (2007), three different albedos $\left(R_{\text {cld }}\right)$ are calculated with the SW code for the cloudy portion of the gridpoint (the subscript "cld" is generally dropped hereafter for simplicity): (1) albedos using the $\bar{\tau}$ and $\bar{r}_{e}$ values of the gridbox (the PPH albedo $R_{\mathrm{PPH}}$ ); (2) albedos using the 1-D histogram of $\tau$ and the gridbox mean value of effective radius $\bar{r}_{e}$ (type 1 ICA albedo $R_{\mathrm{ICA} 1}$ ), i.e., obtained from multiple albedo calculations weighted by the relative frequency in each $\tau$ bin; and (3) albedos using the 2-D histogram (type 2 ICA albedo $R_{\mathrm{ICA} 2}$ ), i.e., obtained from multiple albedo calculations weighted by the relative frequency in each $\left(\tau, r_{e}\right)$ bin. The albedo calculated from the first method minus that calculated from the second gives the classic planeparallel albedo bias with constant microphysics $\left(B_{1}^{R}>0\right)$. The albedo calculated from the first method minus that calculated from the third gives the albedo bias due to joint horizontal variations of $\tau$ and $r_{e}\left(B_{2}^{R}>0\right)$. Both biases correspond to the cloudy portion of the gridpoint only and were carried out separately for each phase. Mathematically, the biases can be expressed as follows:

$$
\begin{aligned}
B_{1}^{R}\left(\bar{\tau}, \bar{r}_{e}, v_{\tau}, \mu_{0}\right)= & R_{\mathrm{PPH}}-R_{\mathrm{ICA} 1} \\
\equiv & \boldsymbol{R}\left(\bar{\tau}, \bar{r}_{e}, \mu_{0}\right) \\
& -\int \boldsymbol{R}\left(\tau, \bar{r}_{e}, \mu_{0}\right) p(\tau) d \tau
\end{aligned}
$$

$$
\begin{aligned}
B_{2}^{R}\left(\bar{\tau}, \bar{r}_{e}, v_{\tau, r_{e}} \mu_{0}\right)= & R_{\mathrm{PPH}}-R_{\mathrm{ICA} 2} \\
\equiv & \boldsymbol{R}\left(\bar{\tau}, \bar{r}_{e}, \mu_{0}\right) \\
& -\iint \boldsymbol{R}\left(\tau, r_{e}, \mu_{0}\right) p\left(\tau, r_{e}\right) d \tau d r_{e}
\end{aligned}
$$

where $\mu_{0}$ is the cosine of the solar zenith angle, $v$ is a measure of either $\tau$ or joint $\tau-r_{e}$ variability (e.g., a shape parameter of the 1-D probability density function $p(\tau)$ or the 2-D probability density function $p\left(\tau, r_{e}\right)$ ), and $\mathbf{R}$ is the reflectance function (for example, the analytical solution of the two-stream approximation). The dependences of the albedo bias on molecular absorption, Rayleigh scattering, and surface albedo are not explicitly shown in the above equations, so Eqs. (2a) and (2b) strictly correspond to the case of isolated clouds. It should be understood, however, that all these factors (assumed to be homogeneous within the $1^{\circ}$ gridbox) are accounted for in our calculations. Note that the ICA calculations are subject to errors due to discretization in the 1-D and 2-D histograms, but these errors are of random nature. Still, they may result in occasional negative values of bias at small values of $\tau$ where $\mathbf{R}$ depends quasi-linearly on $\tau$, and these are set back to zero whenever they occur. Since ICA albedos are based on 1-D radiative transfer calculations, they also suffer, of course, from errors due to neglect of real-world horizontal photon transfer (e.g., Cahalan et al., 1994b).

Oreopoulos et al. (2007) has shown that the albedo bias and the bias in the top-of-the-atmosphere (TOA) shortwave cloud radiative forcing $\triangle \mathrm{SWCRF}^{\mathrm{TOA}}$ are simply related via:

$$
\begin{aligned}
\Delta \mathrm{SWCRF}^{\mathrm{TOA}}(<0) & \equiv \mathrm{SWCRF}_{\mathrm{PPH}}^{\mathrm{TOA}}-\mathrm{SWCRF}_{\mathrm{ICA}}^{\mathrm{TOA}} \\
& =-A_{c} B^{R} \mu_{0} S_{0}
\end{aligned}
$$

where $S_{0}$ is the incident solar irradiance at TOA.

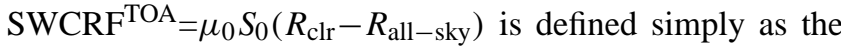
difference in reflected solar fluxes between clear ("clr") and all-sky (i.e., including clear-cloud mixtures) conditions (Ramanathan et al., 1989). To obtain Eq. (3) it is assumed that $R_{\mathrm{all}-\mathrm{sky}}=\left(1-A_{c}\right) R_{\mathrm{clr}}+A_{c} R_{\mathrm{cld}}$. Note that since $B^{R}>0$, $\triangle$ SWCRF $^{\mathrm{TOA}}$ is a negative quantity which does not require separate estimates of the individual PPH and ICA SWCRFs because the identical clear sky fluxes cancel out. With all forcing calculations in this paper refering to TOA, the superscript will be dropped henceforth for simplicity. Moreover, the negative sign of the SWCRF bias is dropped as well, and all magnitude comparisons are discussed in terms of absolute values.

The SWCRF bias estimates are performed separately for each cloud phase (i.e., using each phase's own albedo bias and cloud fraction) for each day of the month in gridboxes where illumination conditions allow MODIS cloud property retrievals, and are then arithmetically averaged to monthly values (we examine the impact of the exact nature of some our averaging choices in Sect. 3.3). Zonal and global averages of the gridpoint monthly values are trivially estimated as in Oreopoulos et al. (2007), but in this case the zero contributions of gridpoints not receiving solar illumination are taken into account in the averages, in contrast to the previous paper where they were ignored. Except for Sect. 3.5 where we explicitly examine Terra-Aqua differences, all other results correspond to averages from the two satellites.

\section{Results}

\subsection{Overpass vs. daily and diurnal SWCRF bias}

The simplest calculation of $\triangle \mathrm{SWCRF}$ for a particular day involves combining the Terra and Aqua PPH albedo bias $B^{R}$ with the insolation corresponding to the gridpoint mean 


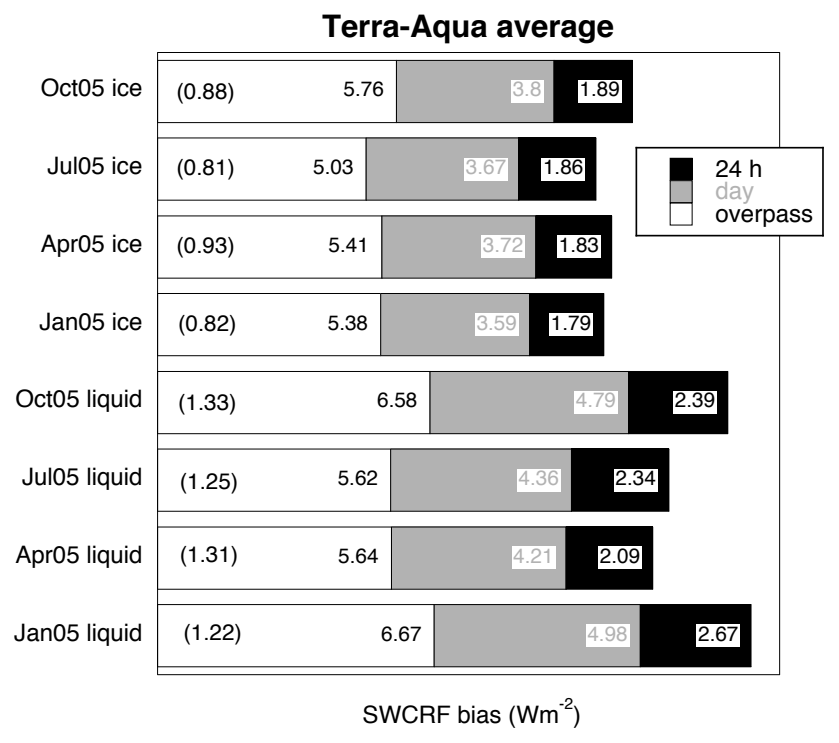

Fig. 1. Stack-bar plot showing the combined MODIS Terra-Aqua global monthly-averaged SWCRF bias using $B_{2}^{R}$ in Eq. (3) for liquid and ice clouds for the four months used in this study. Overpass, daytime, and diurnal (24-h) values are shown (see text). The values in parentheses indicate the ratio of global mean to standard deviation (dispersion) for the overpass case.

SZA for that day as extracted from the MOD08_D3 and MYD08_D3 files. This SZA for most gridpoints corresponds to the SZA of the only daylight overpass for that day and is thus $\sim 90$ min removed from the SZA at local noon. We call the SWCRF bias obtained this way "overpass" $\triangle$ SWCRF. Since it corresponds to relatively high sun conditions it is not a good representation of the true energy impact of the neglect of horizontal cloud inhomogeneity for the duration of the entire day (sunrise to sunset). An accurate true diurnal estimate of $\triangle \mathrm{SWCRF}$ on the other hand is not possible since the diurnal variation of cloud properties (cloud fraction and cloud properties that determine albedo) is not properly resolved with only two available (Terra and Aqua) measurements during daytime. To be able to assess, however, even crudely the influence of varying solar illimination throughout the day, we adopt the methodology of Oreopoulos et al. (2007) for calculating "daytime" $\Delta$ SWCRF's, i.e., pairing the instantaneous $\mathrm{PPH}$ albedo with the instantaneous insolation at 2-h intervals, and integrating over the instances in time with the sun above the horizon. For the time period between sunrise and noon the Terra cloud retrievals are used while from noon to sunset Aqua retrievals are used, both assumed invariant within their respective daytime half. This daytime $\triangle \mathrm{SWCRF}$ is significantly more expensive to compute than the overpass $\triangle \mathrm{SWCRF}$ since it involves multiple bias calculations per day for each gridpoint.

But for the SWCRF biases to be comparable with other biases or forcings that operate uninterrupted (e.g., counterpart thermal infrared CRF biases due to neglect of horizontal cloud condensate or cloud-top temperature variations), the daytime $\triangle$ SWCRF's are not proper measures of the actual energy impact of cloud inomogeneities either. Rather, 24-h ("diurnal") estimates of the SWCRF biases are needed, and those can be obtained (again, as in Oreopoulos et al., 2007) by scaling the daytime biases further by the fraction of the 24-h period with the sun above the horizon for the particular gridpoint.

The mean Terra-Aqua global biases of all three types of $\triangle$ SWCRF due to the combined optical thickness and effective radius horizontal variability (i.e., $B_{2}^{R}$ used in Eq. 3) are shown in stack-bar form in Fig. 1. The values in parentheses indicate the ratio of global mean to standard deviation (dispersion) for the overpass case. The ice cloud $\triangle \mathrm{SWCRF}$ is more spatially variable than that of liquid clouds and there is a slight but distinct tendency of greater dispersion for the vernal and autumnal months compared to the winter and summer months. Due to the seasonal changes in the geographical distribution of the SWCRF bias, the latitudinal dependence of daytime length, and the non-linear nature of the global calculation, an empirical conversion of global overpass bias to global daytime or diurnal bias is not possible: the ratio of daytime to overpass global bias ranges from $\sim 0.65$ to 0.78 , while the ratio of diurnal to overpass global ranges from 0.32 to 0.42 . These values are similar to those of Oreopoulos et al. (2007) for liquid clouds.

Overall, liquid clouds exhibit larger $\triangle \mathrm{SWCRF}$ than ice clouds ( $\sim 6.1$ vs. $5.4 \mathrm{~W} \mathrm{~m}^{-2}$ for overpass bias), with the largest disparity in January $\left(>1 \mathrm{~W} \mathrm{~m}^{-2}\right.$ for overpass bias) and the smallest in April $\left(<0.25 \mathrm{~W} \mathrm{~m}^{-2}\right.$ for overpass bias, increasing interestingly to about $0.5 \mathrm{~W} \mathrm{~m}^{-2}$ for daytime bias). The seasonal variability of bias is relatively stronger for liquid than for ice clouds, especially for daytime and diurnal averages. Further analysis of other aspects of liquid/ice $\triangle$ SWCRF differences is presented in the following subsections.

\subsection{SWCRF bias with and without effective radius variability}

As explained in Oreopoulos et al. (2007) for liquid clouds, inclusion of $r_{e}$ horizontal variability in addition to $\tau$ variability, reduces $\triangle$ SWCRF because of the negative contribution to the PPH bias stemming from the (weak) concavity of the albedo vs. $r_{e}$ curve under constant $\tau$. Essentially, once $\tau$ variability is specified from the combined $\tau-r_{e}$ MODIS retrievals, the $r_{e}$ spatial variability can only generate asymmetry factor and single-scattering albedo variability. This, of course, also applies to ice clouds. Figure 2 contrasts liquid and ice clouds in terms of the global $\triangle \mathrm{SWCRF}$ reduction arising from $r_{e}$ spatial variability contributions, i.e., $\left|A_{c}\left(B_{1}^{R}-B_{2}^{R}\right) \mu_{0} S_{0}\right|$. The global effect of $r_{e}$ spatial variability is a reduction of the combined Terra-Aqua absolute value of the overpass bias by about $0.4 \mathrm{~W} \mathrm{~m}^{-2}(\sim 7 \%)$ for liquid clouds and about $0.25 \mathrm{~W} \mathrm{~m}^{-2}(\sim 5 \%)$ for ice clouds. Other 


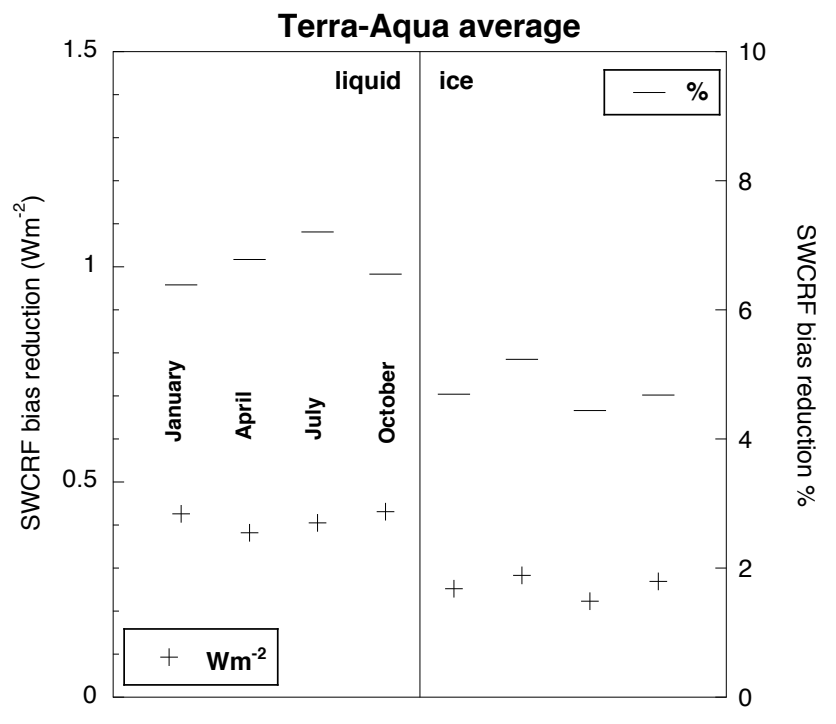

Fig. 2. Absolute $\left(\mathrm{W} \mathrm{m}^{-2}\right)$ and percentage reduction of the combined Terra-Aqua global monthly overpass SWCRF bias from using $B_{2}^{R}$ ( $r_{e}$ spatial variability included in PPH bias calculations) instead of $B_{1}^{R}$ in Eq. (3).

than this, there are no major consequences in the qualitative behavior of $\triangle \mathrm{SWCRF}$ by neglecting $r_{e}$ horizontal inhomogeneity in the calculations. For example, dispersion decreases only very slightly when $r_{e}$ variability is neglected suggesting only minor influences in the spatial patterns of the $\triangle$ SWCRF distribution. Henceforth, all SWCRF bias results will be based on $B_{2}^{R}$ with the understanding that particle size variability is responsible for a smaller than $10 \%$ bias reduction.

\subsection{Cloud fraction and frequency of occurrence contri- butions to the monthly SWCRF bias}

Equation (3) clearly indicates that the daily SWCRF bias of a gridpoint depends on three factors: (1) the PPH albedo bias $B^{R}$ of the cloudy portion of the gridpoint; (2) the cloud fraction $A_{c}$, and (3) the solar irradiance $\mu_{0} S_{0}$ received by the gridpoint. $\triangle \mathrm{SWCRF}$ divided by $A_{c}$ is simply the bias of the reflected TOA flux for the cloudy portion of the gridpoint. For a given incident solar flux, this allows to examine whether high (low) $\triangle$ SWCRF's come from high (low) PPH albedo biases or high (low) cloud fractions or a combination of both. Here, we identify these "per unit cloud fraction" SWCRF biases as "no CF" biases, as in "no cloud fraction was accounted for in the calculation".

Furthermore, when calculating a gridpoint's monthly mean SWCRF bias, averaging can be performed either over the number of days when clouds of a particular phase were indeed observed at the time of the overpass or over the total number of days within the month with an overpass. The latter calculation thus assigns zero contributions to the monthly

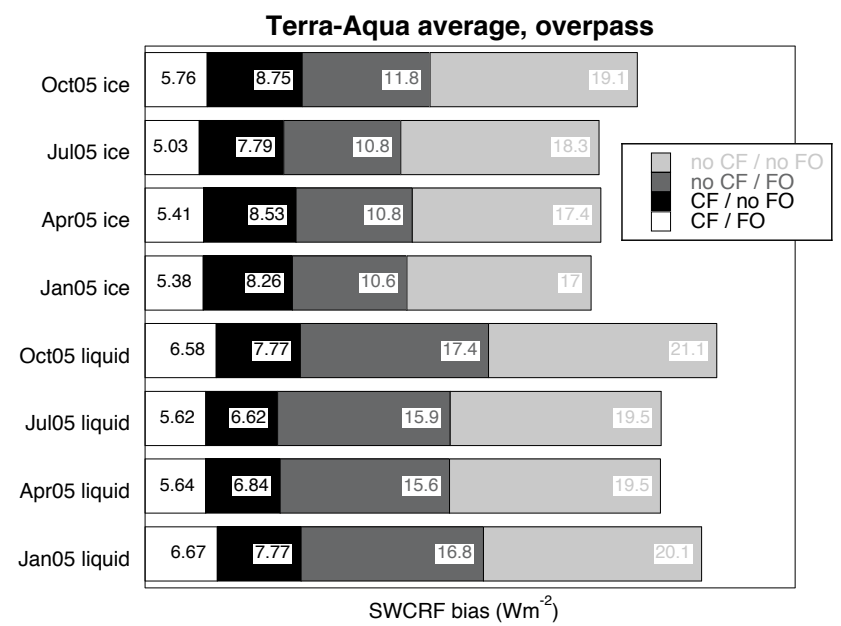

Fig. 3. Stack-bar plot showing global monthly $B_{2}^{R}$-based overpass SWCRF biases for our default calculation (white) and for three other methods that ignore cloud fraction and/or frequency of occurrence of clouds of the respective phase (see text for details). As in the previous plots, the values shown are Terra-Aqua averages.

$\triangle$ SWCRF from days where no cloud of the particular phase was observed. If, for example, for a gridpoint with 25 possible opportunities for observation within a month, only 14 had liquid cloud and therefore allowed estimates of liquid SWCRF bias, a monthly value of $\triangle$ SWCRF can be obtained by dividing either by 25 or by 14 , with the latter calculation reflecting the monthly SWCRF bias of liquid clouds for that gridpoint "when present". This method of not accounting for the frequency of occurrence (FO) of clouds, which obviously gives higher monthly values of $\triangle \mathrm{SWCRF}$, was used by Oreopoulos et al. (2007) and is identified here as the "CF/no FO" method for calculating monthly values of SWCRF bias. With this naming convention the monthly "per unit cloud fraction" SWRCF biases discussed above become the "no CF/FO" biases. Our default choice in this paper (used for the results shown so far and all the results that follow, unless specifically stated otherwise) of including in the averages the zero contributions of days without clouds of a particular phase and accounting for the fraction of the gridpoint that is covered by clouds of a particular phase provides a fairer estimate of monthly SWCRF biases, since the ultimate impact of a forcing (and thererefore its bias) depends on the frequency and spatial extent over which it applies. In this subsection, and whenever a distinction needs to be made between the different flavors of SWCRF bias, we explicitly refer to our default biases as "CF/FO". Finally, one may also be interested in the mean SWCRF bias of the cloudy portion of the gridpoint only for those days when cloud was present in the gridpoint. We call this the "no CF/no FO" SWCRF bias because neither cloud fraction nor frequency of occurrence is accounted for. Such a "SWCRF bias" is more closely associated with the fundamental cloud inhomogeneity characteristics 

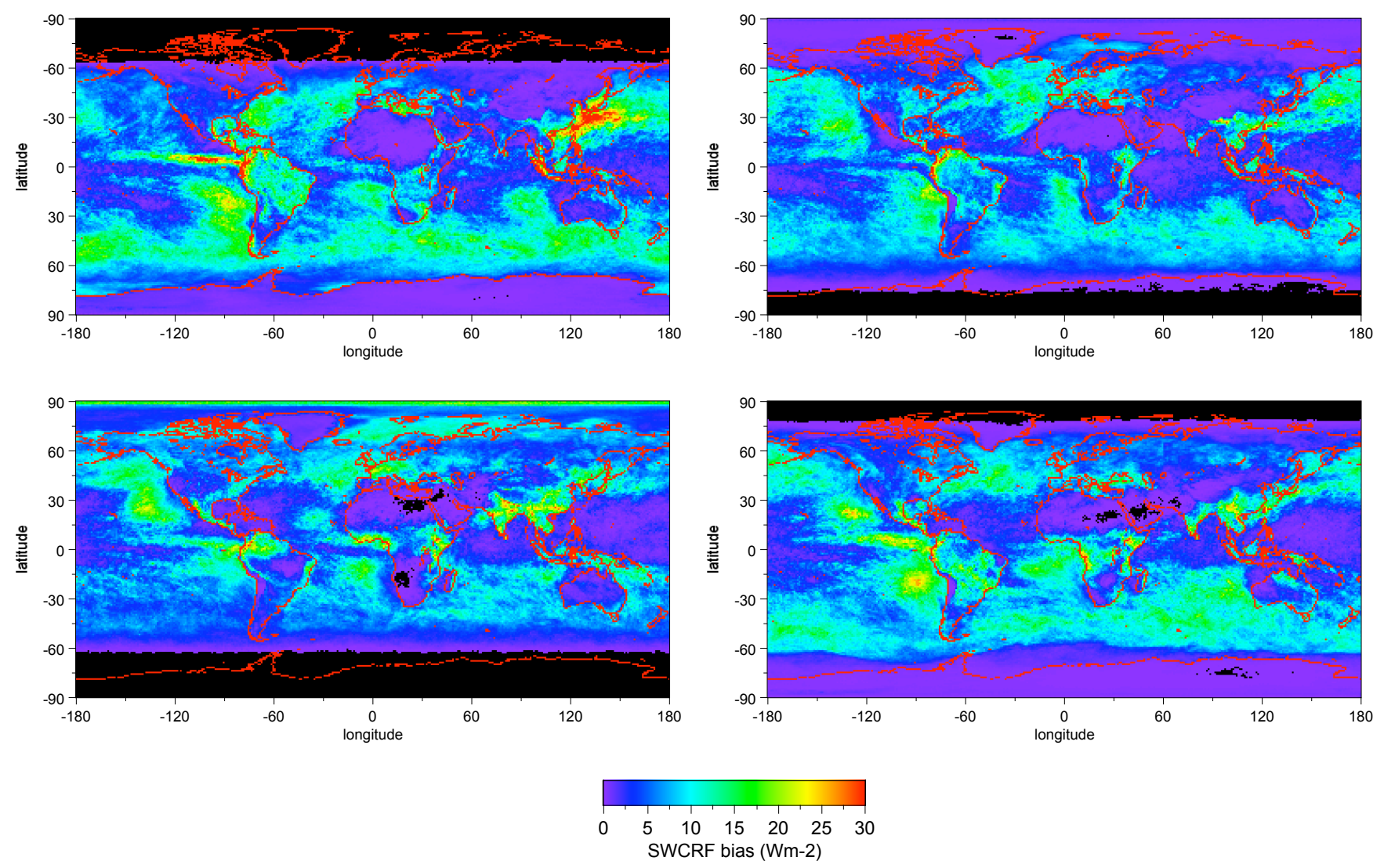

Fig. 4a. Geographical distribution of the combined Terra-Aqua monthly overpass SWCRF bias of liquid clouds from combined optical thickness and effective radius variability for the four months examined in this paper. Black areas indicate no data vailability. Clockwise from top: January 2005, April 2005, October 2005, and July 2005.

driving the plane-parallel albedo bias. Note that further isolation of the contribution of the cloud inhomogeneity characteristics to the SWRCF bias can be achieved by using a globally-averaged insolation everywhere, as in Bennhold and Sherwood (2008), instead of gridpoint-specific $\mu_{0} S_{0}$ values. Since this, however, would essentially be a scaled albedo bias, it would defeat the original purpose of using SWCRF to quantify the PPH bias, i.e., the fact that it is the energetically appropriate quantity.

The global values of all the above types of monthly $\triangle$ SWCRF are compared in the stack bar plot of Fig. 3. The white bars correspond to the same overpass values shown in Fig. 1. Cloud fraction and method of monthly averaging have distinctly different impact on liquid and ice clouds. For instance, if averaging is performed only over the days of the month with clouds of a particular phase present within the gridpoint (black "CF/no FO" bars), the sign of the liquidice $\triangle$ SWCRF difference is reversed with ice clouds now having greater biases than liquid clouds. This means that, when present, ice clouds give overall larger biases than liquid clouds, partly due to larger cloud fractions as will become evident shortly, but their overall monthly $\triangle$ SWCRF is reduced because they occur less frequently. When frequency of cloud occurrence is accounted for (averaging over all days of the month with possible observations), but the bias is normalized by the cloud fraction, i.e., when the reflected flux bias of the cloudy portion is examined, the dominance of liquid over ice SWCRF bias is restored and widened (dark gray "no CF/FO" bars). Evidently, liquid clouds occur more frequently (strictly speaking at the time of the satellite overpass,) and are more inhomogeneous (produce large PPH cloud albedo bias, to be exact) when present. When neither days devoid of clouds of a particular phase nor the cloud fraction is accounted for in monthly estimates of $\triangle \mathrm{SWCRF}$ (light gray "no CF/no FO" bars), the disparity of liquid and ice cloud tapers again because the larger frequency of occurrence of liquid clouds no longer contributes to the monthly bias. Nevertheless, the collective bias in reflected flux for the portions of the gridpoint covered by liquid clouds exceeds that for portions covered by ice clouds, a feature that is most prominent in January and least in July. 

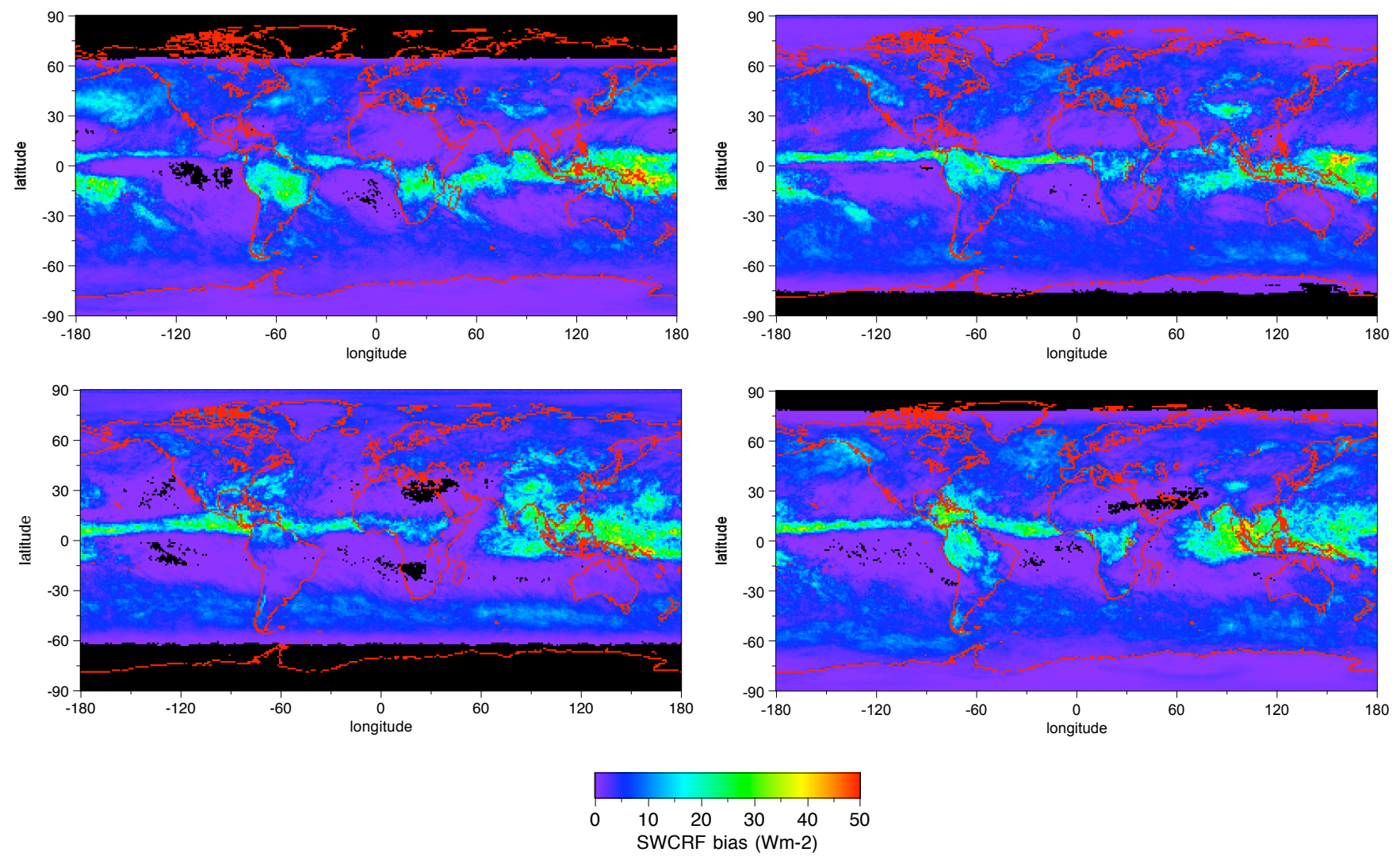

Fig. 4b. As Fig. 4a, but for ice clouds.

\subsection{Geographical distributions of the SWCRF bias}

Figure 4 shows the geographical distribution of liquid and ice cloud overpass SWCRF bias from combined Terra-Aqua results for all four months. The figures reflect known patterns and regimes of liquid and ice clouds and have obvious correlations with cloud inhomogeneity and PPH albedo bias maps in Oreopoulos and Cahalan (2005) and Oreopoulos et al. (2007), respectively (the latter only for liquid clouds). The largest liquid $\triangle$ SWCRFs (Fig. 4a) can be seen in January in the vicinity of the sea of Japan and the Korean peninsula where mid-latitude winter storm systems pass, and in the eastern equatorial Pacific extending to the broader Colombia/Equador region, where cloudiness is due to shallow convection (de Szoeke et al., 2005). Neither of these two regions stands out in the other three months. The marine stratocumulus regions in the eastern parts of the major oceans also exhibit strong seasonality in $\triangle \mathrm{SWCRF}$, with October having in general the largest values, coinciding with seasonal peaks in cloud fraction (Oreopoulos and Davies, 1993). Mid-latitude oceans are quasi-permanent areas of large liquid SWRCF bias, but with values that are largely dependent on available solar illumination (contrast January and July southern oceans). The ice $\triangle$ SWCRF maxima on the other hand are more clearly confined in convective areas and fol- low the movement of the ITCZ (Fig. 4b). The mid-latitude $\triangle$ SWCRF's of ice clouds generally stay below $\sim 15 \mathrm{~W} \mathrm{~m}^{-2}$ and are mostly smaller than their liquid counterparts, but not by as much their color designation suggests, an artifact of the wider bias range covered by the ice cloud colorbar.

The zonal distribution of monthly $\triangle \mathrm{SWCRF}$ (Terra-Aqua averages) is shown only for January and July (Fig. 5). In this case we chose to show the 24-h biases to also capture latitudinal changes in sunlight duration. Features that have previously emerged in the full geographical distribution are prominent, such as the summer peaks in mid-latitude liquid SWCRF bias which assume values close to $7 \mathrm{~W} \mathrm{~m}^{-2}$. The ice $\triangle$ SWCRF peaks are somewhat smaller and appear in the equatorial zone, shifting with the seasonal movement of the ITCZ. Even though plentiful solar illumination is available, minima occur in broad subtropical zones of descending portions of the Hadley cell where deserts and marine stratocumulus regions are encountered. Hemispherical averages are also provided in the figure in order to re-emphasize the strong role of incident solar energy in modulating the SWCRF bias: summer hemispherical values are about twice as large as winter values.

Land-ocean global overpass $\triangle$ SWCRF differences are highlighted in Fig. 6. The bias is clearly greater over oceans for both cloud types and all months with the exception of 


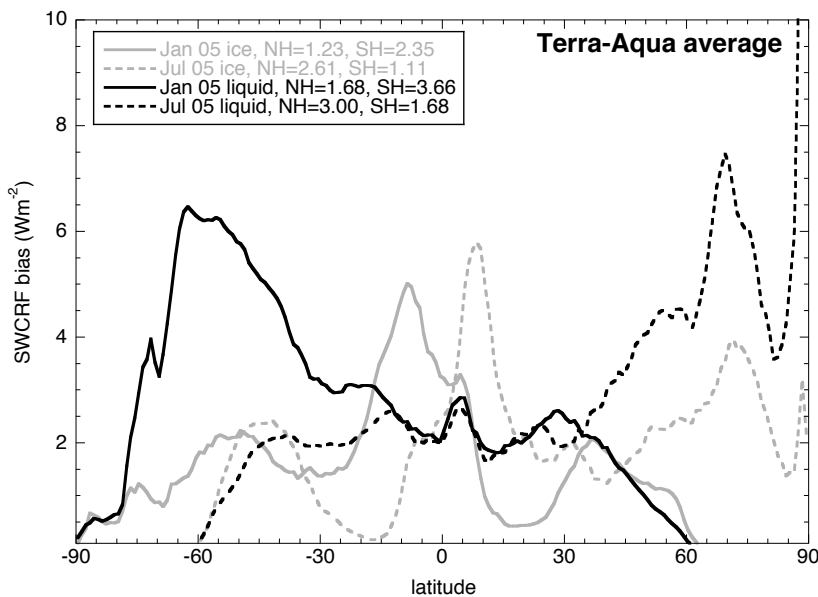

Fig. 5. Zonal variation of the combined Terra-Aqua monthly 24-h SWCRF bias ( $B_{2}^{R}$-based) for January and July 2005 . Hemispheric biases (in $\mathrm{W} \mathrm{m}^{-2}$ ) are also provided in the legend.

July where liquid cloud biases are very similar over land and ocean. The main reason for this seems to be the dramatic decrease of $\triangle \mathrm{SWCRF}$ over the southern midlatitude oceans (Fig. 4a), due to the lower winter insolation. Peaks of $\triangle \mathrm{SWCRF}$ over certain land areas such as over south Asia probably play only minor role in the July near-parity of liquid cloud biases. Besides differences in cloud heterogeneity, cloud fraction, and availability of insolation, another factor for the lower land SWCRF bias is the higher surface albedo of land surfaces which reduces the relative contribution of cloud albedo to the TOA albedo, damping therefore albedo differences between homogeneous and heterogeneous clouds.

\subsection{Terra vs. Aqua differences}

Figure 7 shows the percentage differences (normalized by the combined Terra-Aqua SWCRF bias) of Terra minus Aqua overpass SWCRF biases. Differences are in general negative (Aqua bias greater in absolute value than Terra bias), but this is much more pronounced for ice clouds, suggesting a stronger afternoon cloud inhomogeneity for this type of clouds. Liquid clouds bias differences are either near-zero (April and July) or of opposite sign (January and October). To isolate the morning-afternoon differences in cloud inhomogeneity from cloud fraction and frequency of cloud occurrence contributions, the "no CF/no FO (see Sect. 3.3) overpass $\triangle$ SWCRF relative differences are also plotted. These differences are now always negative for the liquid clouds too. Clearly, cloud fraction and frequency of occurrence reduces morning-afternoon differences due to cloud inhomogeneity alone, i.e., similar to ice clouds, liquid clouds tend also to be more heterogeneous in the afternoon. This is consistent with the cloud inhomogeneity factor results presented

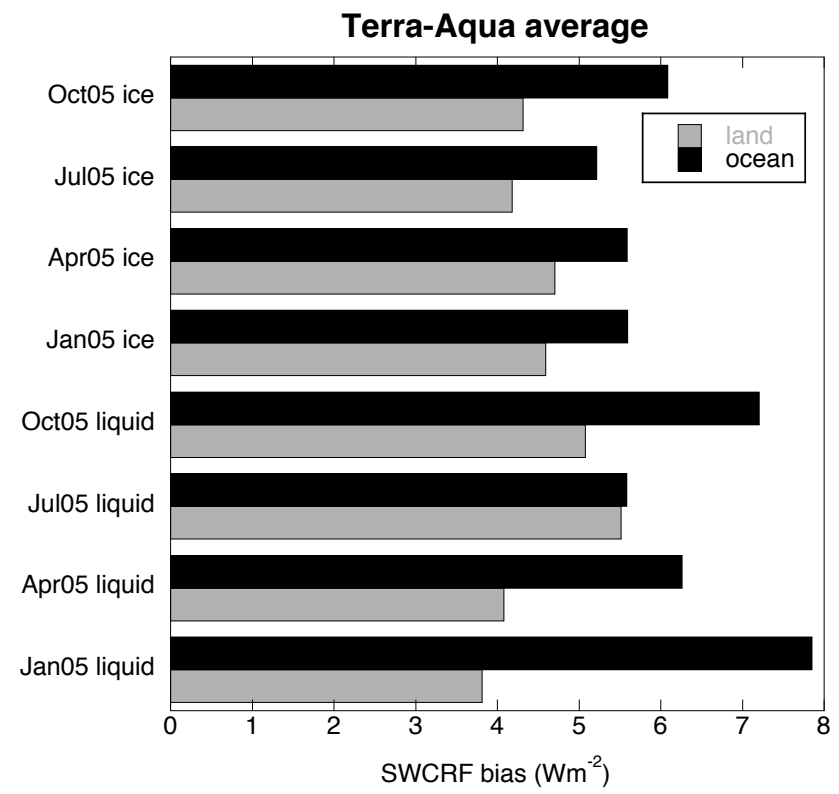

Fig. 6. Monthly combined Terra-Aqua $B_{2}^{R}$-based overpass SWCRF bias averaged separately over the globe's land and ocean gridpoints.

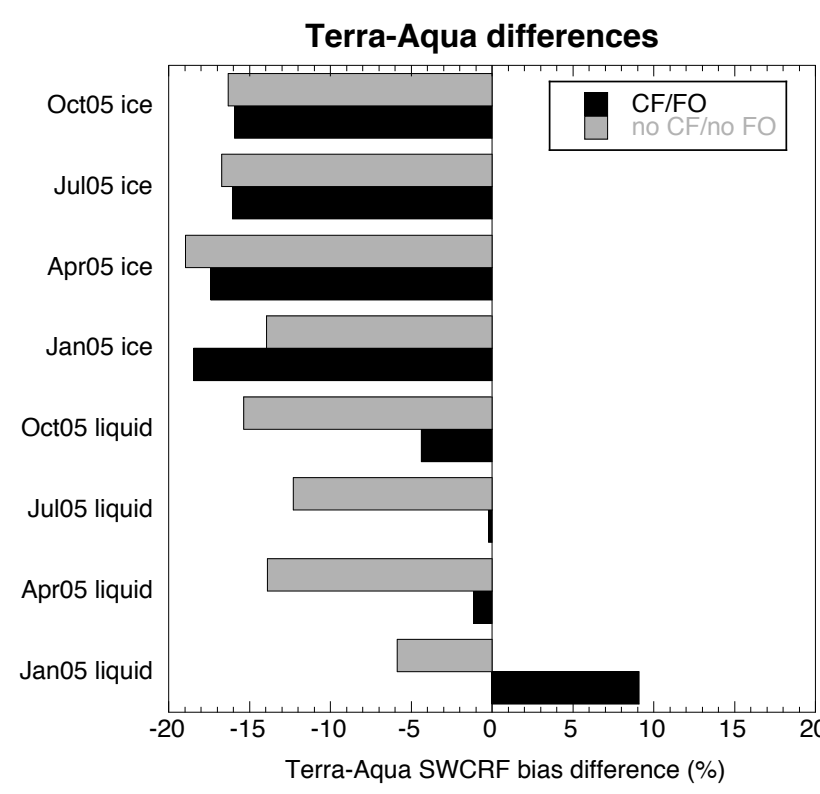

Fig. 7. Percentage difference (normalized by their combined value) of Terra minus Aqua global monthly overpass SWCRF biases $\left(B_{2}^{R}\right.$ based). Along with the default regular ("CF/ FO") SWCRF biases, results from the "no CF/no FO" (see Sect. 3.3) bias calculation are also shown. These reveal the extent to which the Terra-Aqua SWCRF bias differences are due to differences in cloud fraction (CF) and frequency of cloud occurrence (FO).

by Oreopoulos and Cahalan (2005) and the PPH albedo bias results of Oreopoulos et al. (2007).

To further understand Terra and Aqua $\triangle$ SWCRF differences that may be concealed in the global means, we 

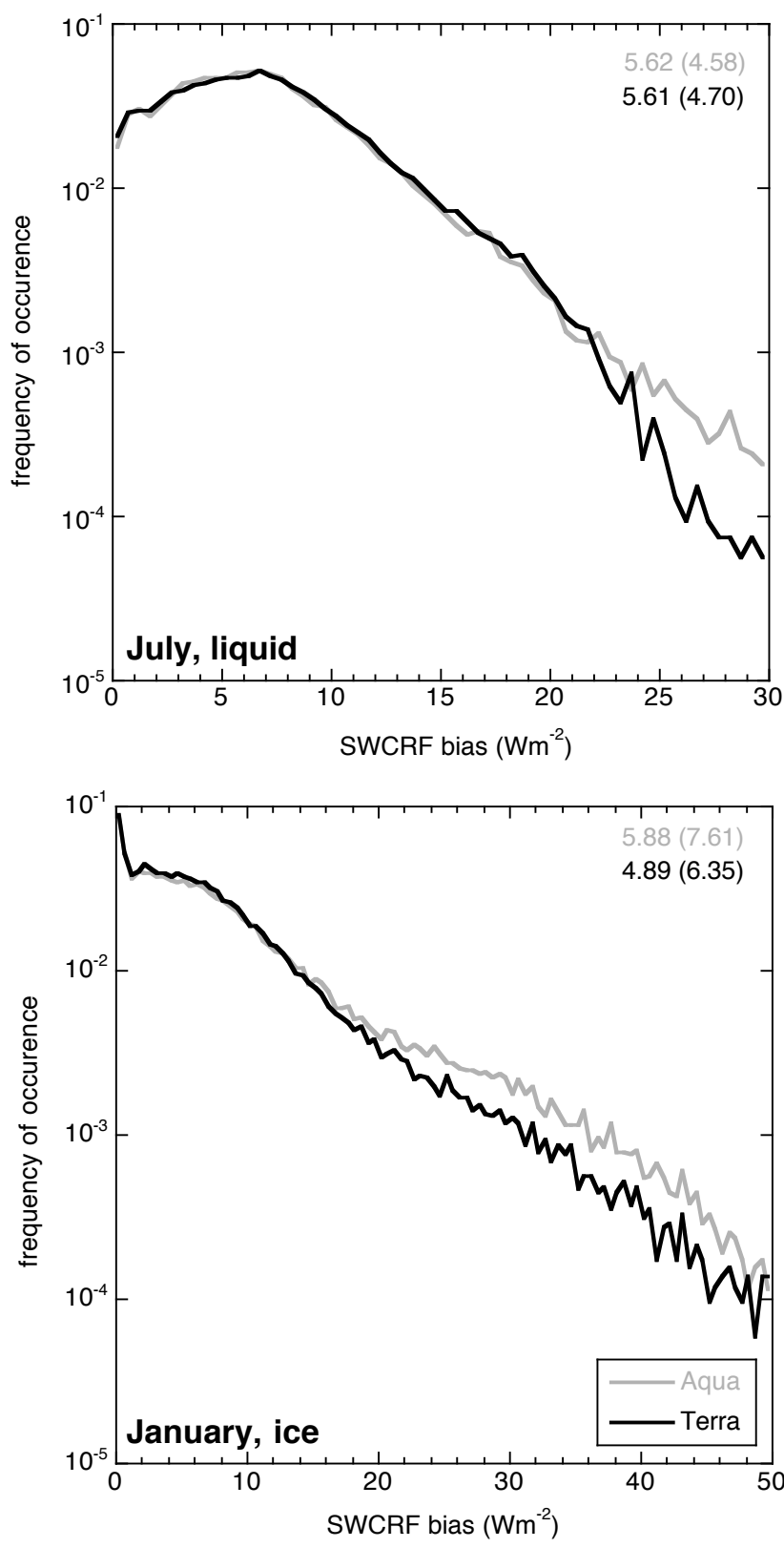

Fig. 8. Normalized logarithmic frequency of occurrence of monthly overpass SWCRF biases ( $B_{2}^{R}$-based) for July 2005 liquid clouds (top) and January 2005 ice clouds (bottom). The global mean SWCRF biases and their standard deviations (in parentheses) are also given.

performed additional analysis on the July 2005 liquid case exhibiting near-zero $\triangle$ SWCRF difference and the January 2005 ice case exhibiting the greatest negative bias difference. Figure 8 plots frequency distributions of Terra and Aqua $\triangle \mathrm{SWCRF}$ for these cases. It is apparent that the near parity of Terra and Aqua July liquid $\triangle \mathrm{SWCRF}$ is not the result of cancellations from different segments of the bias distribution. The Aqua and Terra bias histograms overlap

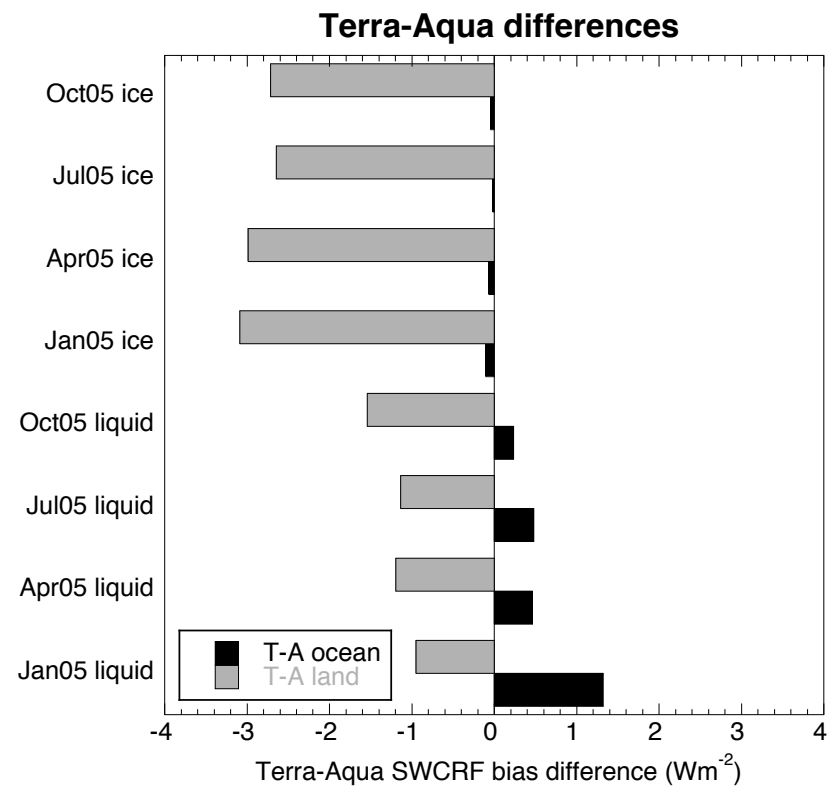

Fig. 9. Terra minus Aqua differences of monthly overpass SWCRF biases ( $B_{2}^{R}$-based) averaged separately over the globe's ocean and land gridpoints.

almost perfectly before starting to diverge only at the highend tail of the distribution corresponding to rare occurences of large $\triangle$ SWCRF values (top panel). On the other hand, for the January ice case, histogram divergence commences at higher normalized frequencies (even though the separation point is again around the $20 \mathrm{~W} \mathrm{~m}^{-2}$ bin as in the liquid case). Aqua forcing biases for this case are not only overall greater, but their distribution is wider as evidenced both by the shape of the histogram and the magnitude of the standard deviation of the bias distribution (in parentheses) which is about $20 \%$ larger than Terra.

Finally, we also examined whether ocean-land contrasts exist in the Terra-Aqua $\triangle \mathrm{SWCRF}$ differences. Figure 9 sheds light on how the global differences of Fig. 7 are ultimately determined. For liquid clouds, Terra $\triangle \mathrm{SWCRF}$ absolute values systematically exceed (fall behind) those of Aqua over ocean (over land); for ice clouds $\triangle$ SWCRF differences are negligible over ocean and quite substantial over land, making the latter responsible for the negative global differences in Fig. 7. That this behaviour is driven almost exclusively by morning-afternoon differences in cloud inhomogeneity between land and ocean was confirmed by plotting the counterpart of Fig. 9 for "no $\mathrm{CF} /$ no FO" $\triangle$ SWCRF's (not shown): oceanic differences hovered around zero while continental differences were strongly negative (Aqua $\triangle$ SWCRF's larger in absolute magnitude), for clouds of both phases. 


\section{Summary and conclusions}

The global plane-parallel bias of the shortwave cloud radiative forcing SWCRF (also known as the shortwave cloud radiative effect) at 1 degree scales is examined using global MODIS (Terra and Aqua) cloud optical property retrievals for four months of 2005 representative of the meteorological seasons, in conjunction with a broadband shortwave radiative transfer code. The absolute value of the (negative) global SWCRF bias of liquid clouds at the top of the atmosphere is $\sim 6 \mathrm{~W} \mathrm{~m}^{-2}$ for MODIS overpass times skewed towards near solar noon insolations, while the SWCRF bias for ice clouds is smaller in absolute terms by $\sim 0.7 \mathrm{~W} \mathrm{~m}^{-2}$, but with stronger spatial variability. A significant contributor to the greater liquid cloud SWCRF biases is the higher frequency of occurrence of liquid clouds, which when combined with the higher average plane-parallel albedo bias, overcompensate for the higher cloud fraction of ice clouds, when present. If effective radius variability is neglected and only optical thickness horizontal variations are accounted for, SWCRF biases increase by about $0.3-0.4 \mathrm{~W} \mathrm{~m}^{-2}$ on average. Rough conversions of these biases to daytime and diurnal (24-h) values yield values that are $\sim 25-35 \%$ and $\sim 60$ $70 \%$ smaller, respectively. Oceanic clouds of both phases exhibit larger (more negative) SWCRF biases than continental clouds. Finally, morning (Terra)-afternoon (Aqua) differences in SWCRF bias are much more pronounced for ice than liquid clouds, reaching about $\sim 15 \%$ (Aqua producing stronger negative bias) on global scales, with almost all contribution to the difference coming from land areas.

If one wants to distill the analysis presented here to a single representative number of the lower limit of global SWCRF bias, the diurnal " $24 \mathrm{~h}$ " values of Fig. 1, corresponding to combined optical thickness and effective radius variability and accounting for both cloud fraction and frequency of occurrence, should probably be chosen. Taking the arithmetic mean of the four monthly values yields a SWCRF bias of $2.4 \mathrm{~W} \mathrm{~m}^{-2}$ for liquid clouds and $1.8 \mathrm{~W} \mathrm{~m}^{-2}$ for ice clouds. Due to the nature of MODIS observations where liquid and ice clouds cover non-overlapping portions of the gridpoint, these numbers must be added. Their total of $4.2 \mathrm{~W} \mathrm{~m}^{-2}$ can then be considered a lower bound estimate of global SWCRF bias. The characterization of this value as a lower bound is justified by the inclusion of zero contributions from cloudfree and non-illuminated areas, and to a lesser extent by the omission of the (relatively small) number of clouds classified by MODIS as having an "undetermined" phase. Still, a more accurate assessment requires knowledge of the full diurnal variation of cloud properties, and perhaps more sophisticated treatments of atmospheric (e.g., accounting for aerosols) and surface albedo effects.

Our global SWCRF bias values, along with the more detailed breakdown of bias behaviour revealed in our full suite of results should provide a valuable validation reference point for global modeling approaches that are able to gen- erate mesoscale cloud inhomogeneity, provided that some effort is extended to simulate the MODIS worldview. This would ideally entail use of some type of "MODIS simulator" where the most obvious limitations of passive radiometry retrievals are emulated. Such limitations include, but are not restricted to, views of low clouds only when not obscured by higher clouds, optical thickness integration over the entire atmospheric column, and strong dependence of cloud microphysics and phase characterization to near cloud top conditions. The simulator should also encompass the temporal and spatial sampling strategies of MODIS. Furthermore, it is important to keep in mind that any calculations of planeparallel albedo or forcing bias are tied to the spatial scale at which the horizontal variability of cloud properties is considered (see Oreopoulos and Davies, 1998, for dependences on spatial scale), which means that any global model-MODIS comparison should be performed on identical grids. This would require that the global model can be run at a resolution of at least $1^{\circ}$, something probably within the capabilities of many modeling groups today. A further condition to make such a validation meanigful is that the cloud scheme which generates subgrid cloud variability explicitly or implicitly resolves cloud fields at approximate the same scale $(\sim 1 \mathrm{~km})$ as the resolution of the cloud optical property retrievals used to build the MODIS Level-3 statistics.

Acknowledgements. L. Oreopoulos gratefully acknowledges support from the US Department of Energy, Office of Science, Office of Biological and Environmental Research, Environmental Sciences Division as part of the ARM program under grant DE-FG02-07ER64354. Partial funding to all GSFC authors was also provided by the NASA Radiation Sciences Program.

Edited by: A. Nenes

\section{References}

Barker, H. W.: A parameterization for computing grid-averaged solar fluxes for inhomogeneous marine boundary layer clouds, part I: methodology and homogeneous biases, J. Atmos. Sci., 53, 2289-2303, 1996.

Baum, B. A., Heymsfield A., Yang, P., and Bedka, S. T.: Bulk scattering properties for the remote sensing of ice clouds I: Microphysical data and models, J. Appl. Meteor., 44, 1885-1895, 2005.

Bennhold, F. and Sherwood, S.: Erroneous Relationships among Humidity and Cloud Forcing Variables in Three Global Climate Models, J. Climate, 21, 4190-4206, 2008.

Cahalan, R. F., Ridgway, W., Wiscombe, W. J., Bell, T. L., and Snider, J. B.: The albedo of fractal stratocumulus clouds, J. Atmos. Sci., 51, 2434-2455, 1994a.

Cahalan, R. F., Ridgway, W., Wiscombe, W. J., Harshvardhan, and Gollmer, S.: Independent pixel and Monte Carlo estimates of stratocumulus albedo, J. Atmos. Sci., 51, 3776-3790, 1994 b.

Chou, M.-D., Suarez, M. J., Ho, C.-H., Yan, M. M.-H., and Lee, K.-T.: Parameterizations for cloud overlapping and shortwave 
single-scattering properties for use in general circulation and cloud ensemble models, J. Climate, 11, 202-214, 1998.

de Szoeke, S. P., Bretherton, C. S., Bond, N. A., Cronin, M. F., and Morley, B. M.: EPIC $95^{\circ} \mathrm{W}$ Observations of the Eastern Pacific Atmospheric Boundary Layer from the Cold Tongue to the ITCZ, J. Atmos. Sci., 62, 426-442, 2005.

Derber, J. C., Parrish, D. F., and Lord, S. J.: The new global operational analysis system at the National Meteorological Center, Weath. Forec., 6, 538-547, 1991.

$\mathrm{Fu}, \mathrm{Q}$.: An accurate parameterization of the solar radiative properties of cirrus clouds for climate models, J. Climate, 9, 20582082, 1996.

Hu, Y. X. and Stamnes, K.: An accurate parameterization of the radiative properties of water clouds suitable for use in climate models, J. Climate, 6, 728-742, 1993.

Khairoutdinov, M., Randall, D. A., and DeMott, C.: Simulations of the Atmospheric General Circulation Using a Cloud-Resolving Model as a Superparameterization of Physical Processes, J. Atmos. Sci., 62, 2136-2154, 2005.

King, M. D., Menzel W. P., Kaufman Y. J., Tanré D., Gao, B.-C., Platnick, S. E., Ackerman, S. A., Remer, L. A., Pincus R., and Hubanks, P. A.: Cloud and aerosol properties, precipitable water, and profiles of temperature and water vapor from MODIS, IEEE Trans. Geosc. Rem. Sens., 41, 442-458, 2003.

Moody, E. G., King, M. D, Platnick, S. E., Schaaf, C. B., and Gao, F.: Spatially complete global spectral surface albedos: Valueadded datasets derived from Terra MODIS land products, IEEE Trans. Geosci. Remote Sens., 43, 144-158, 2005.

Oreopoulos, L. and Davies, R.: Statistical dependence of albedo and cloud cover on sea surface temperature for two tropical marine stratocumulus regions, J. Climate, 6, 2434-2447, 1993.
Oreopoulos, L. and Davies, R.: Plane parallel albedo biases from satellite observations. Part I: Dependence on resolution and other factors, J. Climate, 11, 919-932, 1998.

Oreopoulos, L. and Cahalan, R. F.: Cloud inhomogeneity from MODIS, J. Climate, 18, 5110-5124, 2005.

Oreopoulos, L., Cahalan R. F., and Platnick, S.: The plane-parallel albedo bias of liquid clouds from MODIS observations, J. Climate, 20, 5114-5125, 2007.

Oreopoulos, L. and Platnick, S.: The radiative susceptibility of cloudy atmospheres to droplet number perturbations, 2: Global analysis from MODIS. J. Geophys. Res., 113, D14S21, doi:10.1029/2007JD009655, 2008.

Pincus, R., McFarlane, S. A., and Klein, S. A.: Albedo bias and the horizontal variability of clouds in subtropical marine boundary layers: observations from ships and satellites, J. Geophys. Res., 104, 6183-6191, 1999.

Ramanathan, V., Ahmad E., Cess R. D., Harrison, E. F., Minnis, P., Barkstrom, B. R., and Hartmann, D.: Cloud-radiative forcing and climate: Results from the earth radiation budget experiment, Science, 243, 57-63, 1989.

Rossow, W. B., Delo C., and Cairns, B.: Implications of the observed mesoscale variations of clouds for the Earth's radiation budget, J. Climate, 15, 557-585, 2002.

Yang, P., Liou, K. N., Wyser, K., and Mitchell, D.: Parameterization of the scattering and absorption properties of individual ice crystals, J. Geophys. Res., 105, 4699-4718, 2000.

Yang, P., Wei, H., Huang H.-L., Baum B. A., Hu, Y. X., Kattawar, G. W., Mishchenko, M. I., and Fu, Q.: Scattering and absorption property database for nonspherical ice particles in the nearthrough far-infrared spectral region, Appl. Optics, 44, 55125523, 2005. 